\title{
Axiomatic Characterizations of IVF Rough Approximation Operators
}

\author{
Guangji Yu \\ School of Information and Statistics, Guangxi University of Finance and Economics, Nanning, Guangxi 530003, China \\ Correspondence should be addressed to Guangji Yu; guangjiyu100@126.com
}

Received 11 May 2013; Revised 13 January 2014; Accepted 13 January 2014; Published 31 March 2014

Academic Editor: Gerhard-Wilhelm Weber

Copyright (C) 2014 Guangji Yu. This is an open access article distributed under the Creative Commons Attribution License, which permits unrestricted use, distribution, and reproduction in any medium, provided the original work is properly cited.

This paper is devoted to the study of axiomatic characterizations of IVF rough approximation operators. IVF approximation spaces are investigated. The fact that different IVF operators satisfy some axioms to guarantee the existence of different types of IVF relations which produce the same operators is proved and then IVF rough approximation operators are characterized by axioms.

\section{Introduction}

Rough set theory was proposed by Pawlak $[1,2]$ as a mathematical tool for data reasoning. It may be seen as an extension of classical set theory; it has been proved to be an effective approach to deal with intelligent systems characterized by insufficient and incomplete information and has been successfully applied to machine learning, intelligent systems, inductive reasoning, pattern recognition, mereology, image processing, signal analysis, knowledge discovery, decision analysis, expert systems, and many other fields.

There are mainly two approaches to the development of rough set theory. One is the constructive approach in which rough approximation operators are constructed by means of relations, partitions, coverings, neighborhood systems, and so on. The constructive approach is suitable for practical applications of rough sets. The other one is the axiomatic approach. In this approach, axioms sets are used to characterize rough approximation operators that guarantee the existence of certain types of relations which produce the same operators. This approach is appropriate for studying algebra structures of rough sets. Under this point of view, rough set theory may be interpreted as an extension of set theory with two additional unary operators.

As a generalization of Zadeh's fuzzy set, interval-valued fuzzy (IVF, for short) sets were introduced by Gorzalczany [3] and Türksen [4], and they were applied to the fields of approximate inference, signal transmission, and controller.
By integrating Pawlak rough set theory with IVF set theory, Sun et al. [5] introduced IVF rough sets based on an IVF approximation space, defined IVF information systems, and discussed their attribute reduction. Gong et al. [6] studied the knowledge discovery in IVF information systems. Zhang et al. [7] discussed $(\mathscr{I}, \mathscr{T})$-IVF rough sets based on an IVF approximation space on two universes of discourse.

The purpose of this paper is to investigate IVF rough sets by using axiomatic approaches and to give axiomatic characterizations of IVF rough approximation operators.

\section{Preliminaries}

Throughout this paper, "interval-valued fuzzy" denotes briefly by "IVF." $U$ denotes a nonempty finite set called the universe of discourse. $I$ denotes $[0,1]$ and $[I]$ denotes $\{[a, b]$ : $a, b \in I$ and $a \leq b\} . \mathscr{P}(U)$ denotes the family of all subsets of $U . \bar{a}$ denotes $[a, a]$ for each $a \in[0,1]$.

For any $\left[a_{j}, b_{j}\right] \in[I](j=1,2)$, we define the following:

$$
\begin{aligned}
& {\left[a_{1}, b_{1}\right]=\left[a_{2}, b_{2}\right] } \Longleftrightarrow a_{1}=a_{2}, \quad b_{1}=b_{2} ; \\
& {\left[a_{1}, b_{1}\right] \leq\left[a_{2}, b_{2}\right] } \Longleftrightarrow a_{1} \leq a_{2}, \quad b_{1} \leq b_{2} ; \\
& {\left[a_{1}, b_{1}\right]<\left[a_{2}, b_{2}\right] } \Longleftrightarrow\left[a_{1}, b_{1}\right] \\
& \leq\left[a_{2}, b_{2}\right], \quad\left[a_{1}, b_{1}\right] \neq\left[a_{2}, b_{2}\right] ; \\
& \overline{1}-\left[a_{1}, b_{1}\right] \quad \text { or }\left[a_{1}, b_{1}\right]^{c}=\left[1-b_{1}, 1-a_{1}\right] .
\end{aligned}
$$

Obviously, $\left([a, b]^{c}\right)^{c}=[a, b]$ for each $[a, b] \in[I]$. 
Definition 1 (see $[3,4]$ ). For any $\left\{\left[a_{j}, b_{j}\right]: j \in J\right\} \subseteq[I]$, we define the following:

$$
\bigvee_{j \in J}\left[a_{j}, b_{j}\right]=\left[\bigvee_{j \in J} a_{j}, \bigvee_{j \in J} b_{j}\right] \quad \bigwedge_{j \in J}\left[a_{j}, b_{j}\right]=\left[\bigwedge_{j \in J} a_{j}, \bigwedge_{j \in J} b_{j}\right],
$$

where $\bigvee_{j \in J} a_{j}=\sup \left\{a_{j}: j \in J\right\}$ and $\bigwedge_{j \in J} a_{j}=\inf \left\{a_{j}: j \in\right.$ $J\}$.

Definition 2 (see $[3,4]$ ). An IVF set $A$ in $U$ is defined by a mapping $A: U \rightarrow[I]$. Denote that

$$
A(x)=\left[A^{-}(x), A^{+}(x)\right], \quad x \in U .
$$

Then $A^{-}(x)$ (resp., $\left.A^{+}(x)\right)$ is called the lower (resp., upper) degree to which $x$ belongs to $A$. $A^{-}$(resp., $A^{+}$) is called the lower (resp., upper) IVF set of $A$.

The set of all IVF sets in $U$ is denoted by $F^{(i)}(U)$.

Let $a, b \in I . \widetilde{[a, b]}$ represents the IVF set which satisfies $\widetilde{[a, b]}(x)=[a, b]$ for each $x \in U$. We denoted $\widetilde{[a, a]}$ by $\tilde{a}$.

We recall some basic operations on $F^{(i)}(U)$ as follows $([3,4])$ : for any $A, B \in F^{(i)}(U)$ and $[a, b] \in[I]$, consider the following.
(1) $A=B \Leftrightarrow A(x)=B(x)$ for each $x \in U$.
(2) $A \subseteq B \Leftrightarrow A(x) \leq B(x)$ for each $x \in U$.
(3) $A=B^{c} \Leftrightarrow A(x)=B(x)^{c}$ for each $x \in U$.
(4) $(A \cap B)(x)=A(x) \wedge B(x)$ for each $x \in U$.
(5) $(A \cup B)(x)=A(x) \vee B(x)$ for each $x \in U$.

Moreover,

$$
\left(\bigcup_{j \in J} A\right)(x)=\bigvee_{j \in J} A(x), \quad\left(\bigcap_{j \in J} A\right)(x)=\bigwedge_{j \in J} A(x),
$$

where $\left\{A_{j}: j \in J\right\} \subseteq F^{(i)}(U)$.

(6) $([a, b] A)(x)=[a, b] \wedge\left[A^{-}(x), A^{+}(x)\right]$ for each $x \in U$.

Definition 3 (see [8]). $A \in F^{(i)}(U)$ is called an IVF point in $U$, if there exist $[a, b] \in[I]-\{\overline{0}\}$ and $x \in U$ such that

$$
A(y)= \begin{cases}{[a, b],} & y=x \\ 0, & y \neq x .\end{cases}
$$

We denote $A$ by $x_{[a, b]}$.

Remark 4. Consider $A=\bigcup_{x \in U} A(x) x_{\overline{1}}\left(A \in F^{(i)}(U)\right)$.

\section{IVF Rough Approximation Operators and IVF Rough Sets}

Recall that $R$ is called an IVF relation on $U$ if $R \in F^{(i)}(U \times U)$.

Definition 5 (see [5]). Let $R$ be an IVF relation on $U$. Then $R$ is called the following.
(1) Serial, if $\bigvee_{y \in U} R(x, y)=\overline{1}$ for each $x \in U$.

(2) Reflexive, if $R(x, x)=\overline{1}$ for each $x \in U$.

(3) Symmetric, if $R(x, y)=R(y, x)$ for any $x, y \in U$.

(4) Transitive, if $R(x, z) \geq R(x, y) \wedge R(y, z)$ for any $x, y, z \in U$.

Let $R$ be an IVF relation on $U$. $R$ is called preorder if $R$ is reflexive and transitive ([9]).

Definition 6 (see [5]). Let $R$ be an IVF relation on $U$. The pair $(U, R)$ is called an IVF approximation space. The IVF lower and the IVF upper approximation of $A \in F^{(i)}(U)$ with respect to $(U, R)$, denoted by $\underline{R}(A)$ and $\bar{R}(A)$, are, respectively, defined as follows:

$$
\begin{gathered}
\underline{R}(A)(x)=\bigwedge_{y \in U}(A(y) \vee(\overline{1}-R(x, y))) \quad(x \in U), \\
\bar{R}(A)(x)=\bigvee_{y \in U}(A(y) \wedge R(x, y)) \quad(x \in U) .
\end{gathered}
$$

The pair $(\underline{R}(A), \bar{R}(A))$ is called the IVF rough set of $A$ with respect to $(U, R)$.

$\underline{R}: F^{(i)}(U) \rightarrow F^{(i)}(U)$ and $\bar{R}: F^{(i)}(U) \rightarrow F^{(i)}(U)$ are called the IVF lower approximation operator and the IVF upper approximation operator, respectively. In general, we refer to $\underline{R}$ and $\bar{R}$ as the IVF rough approximation operators.

Remark 7. Let $(U, R)$ be an IVF approximation space. Then we get the following.

(1) For any $x, y \in U$,

$$
\bar{R}\left(x_{\overline{1}}\right)(y)=R(y, x), \quad \underline{R}\left(\left(x_{\overline{1}}\right)^{c}\right)(y)=\overline{1}-R(y, x) .
$$

(2) For each $[a, b] \in[I], \underline{R}(\widetilde{[a, b]}) \supseteq \widetilde{[a, b]} \supseteq \bar{R}(\widetilde{[a, b]})$.

Proposition 8 (see [5]). Let $(U, R)$ be an IVF approximation space. Then for each $A \in F^{(i)}(U)$,

$$
\begin{aligned}
& (\underline{R}(A))^{-}=\underline{R^{+}}\left(A^{-}\right), \quad(\underline{R}(A))^{+}=\underline{R^{-}}\left(A^{+}\right), \\
& (\bar{R}(A))^{-}=\overline{R^{-}}\left(A^{-}\right), \quad(\bar{R}(A))^{+}=\overline{R^{+}}\left(A^{+}\right) .
\end{aligned}
$$

Theorem 9. Let $(U, R)$ be an IVF approximation space. Then for any $A, B \in F^{(i)}(U),\left\{A_{j}: j \in J\right\} \subseteq F^{(i)}(U)$, and $[a, b] \in[I]$, one has the following.

(1) $\underline{R}(\widetilde{1})=\widetilde{1}, \bar{R}(\widetilde{0})=\widetilde{0}$.

(2) $A \subseteq B \Rightarrow \underline{R}(A) \subseteq \underline{R}(B), \bar{R}(A) \subseteq \bar{R}(B)$.

(3) $\underline{R}\left(A^{c}\right)=(\bar{R}(A))^{c}, \bar{R}\left(A^{c}\right)=(\underline{R}(A))^{c}$.

(4) $\underline{R}\left(\bigcap_{j \in J} A_{j}\right)=\bigcap_{j \in J} \underline{R}\left(A_{j}\right), \bar{R}\left(\bigcup_{j \in J} A_{j}\right)=\bigcup_{j \in J} \bar{R}\left(A_{j}\right)$.

(5) $\underline{R}(\widetilde{[a, b]} \cup A)=\widetilde{[a, b]} \cup \underline{R}(A), \bar{R}([a, b] A)=[a, b] \bar{R}(A)$. 
Proof. (1) and (2) are obvious.

(3) $\forall x \in U$,

$$
\begin{aligned}
& \underline{R}\left(A^{c}\right)(x) \\
& =\left[\bigwedge_{y \in U}\left(\left(1-A^{+}(y)\right) \vee\left(1-R^{+}(x, y)\right)\right),\right. \\
& \left.\bigwedge_{y \in U}\left(\left(1-A^{-}(y)\right) \vee\left(1-R^{-}(x, y)\right)\right)\right] \\
& =\left[\bigwedge_{y \in U}\left(1-A^{+}(y) \wedge R^{+}(x, y)\right),\right. \\
& \left.\bigwedge_{y \in U}\left(1-A^{-}(y) \wedge R^{-}(x, y)\right)\right] \\
& =\left[1-\bigvee_{y \in U}\left(A^{+}(y) \wedge R^{+}(x, y)\right),\right. \\
& \left.1-\bigvee_{y \in U}\left(A^{-}(y) \wedge R^{-}(x, y)\right)\right] \\
& =\left[1-(\bar{R}(A))^{+}(x), 1-(\bar{R}(A))^{-}(x)\right] \\
& =\overline{1}-\left[(\bar{R}(A))^{-}(x),(\bar{R}(A))^{+}(x)\right] \\
& =\overline{1}-\bar{R}(A)(x)=(\bar{R}(A))^{c}(x) \text {. }
\end{aligned}
$$

Then $R\left(A^{c}\right)=(\bar{R}(A))^{c}$.

Pick $A=B^{c}$. Since $\underline{R}\left(B^{c}\right)=(\bar{R}(B))^{c}$, we have

$$
\bar{R}\left(A^{c}\right)=\bar{R}(B)=\left((\bar{R}(B))^{c}\right)^{c}=\left(\underline{R}\left(B^{c}\right)\right)^{c}=(\underline{R}(A))^{c} .
$$

(4) $\forall x \in U$,

$$
\begin{aligned}
& \underline{R}\left(\bigcap_{j \in J} A_{j}\right)(x) \\
& =\bigwedge_{y \in U}\left(\left(\bigcap_{j \in J} A_{j}\right)(y) \vee(\overline{1}-R(x, y))\right) \\
& \left.=\bigwedge_{y \in U}\left(\bigwedge_{j \in J} A_{j}(y)\right) \vee(\overline{1}-R(x, y))\right) \\
& =\bigwedge_{y \in U}\left(\bigwedge_{j \in J}\left(A_{j}(y) \vee(\overline{1}-R(x, y))\right)\right)
\end{aligned}
$$

$$
\begin{aligned}
& =\bigwedge_{j \in J}\left(\bigwedge_{y \in U}\left(A_{j}(y) \vee(\overline{1}-R(x, y))\right)\right) \\
& =\bigwedge_{j \in J} \underline{R}(A)(x)=\left(\bigcap_{j \in J} \underline{R}\left(A_{j}\right)\right)(x) .
\end{aligned}
$$

Then $\underline{R}\left(\bigcap_{j \in J} A_{j}\right)=\bigcap_{j \in J} \underline{R}\left(A_{j}\right)$.

By (3) and $\underline{R}\left(\bigcap_{j \in J}\left(A_{j}\right)^{c}\right)=\bigcap_{j \in J} \underline{R}\left(\left(A_{j}\right)^{c}\right)$, we have

$$
\begin{aligned}
\left(\bar{R}\left(\bigcup_{j \in J} A_{j}\right)\right)^{c} & =\underline{R}\left(\left(\bigcup_{j \in J} A_{j}\right)^{c}\right) \\
& =\underline{R}\left(\bigcap_{j \in J} A_{j}^{c}\right)=\bigcap_{j \in J} \underline{R}\left(A_{j}^{c}\right) \\
& =\bigcap_{j \in J}\left(\bar{R}\left(A_{j}\right)\right)^{c}=\left(\bigcup_{j \in J} \bar{R}\left(A_{j}\right)\right)^{c} .
\end{aligned}
$$

Thus $\bar{R}\left(\bigcup_{j \in J} A_{j}\right)=\bigcup_{j \in J} \bar{R}\left(A_{j}\right)$.

(5) $\forall x \in U$,

$$
\begin{aligned}
\bar{R}( & {[a, b] A)(x) } \\
& =\bigvee_{y \in U}(([a, b] A)(y) \wedge R(x, y)) \\
& =\bigvee_{y \in U}(([a, b] \wedge A(y)) \wedge R(x, y)) \\
& =\bigvee_{y \in U}([a, b] \wedge(A(y) \wedge R(x, y))) \\
& =[a, b] \wedge\left(\bigvee_{y \in U}(A(y) \wedge R(x, y))\right) \\
& =[a, b] \wedge \bar{R}(A)(x)=([a, b] \bar{R}(A))(x) .
\end{aligned}
$$

Then $\bar{R}([a, b] A)=[a, b] \bar{R}(A)$.

Similarly,

$$
\underline{R}(\widetilde{[a, b]} \cup A)=\widetilde{[a, b]} \cup \underline{R}(A) .
$$

Theorem 10. Let $(U, R)$ be an IVF approximation space. Then one has the following.

(1) $R$ is serial $\Leftrightarrow\left(I L S E^{\prime}\right) \forall[a, b] \in[I], \underline{R}(\widetilde{[a, b]})=\widetilde{[a, b]}$.

$$
\begin{aligned}
& \Leftrightarrow \frac{\left(I U S E^{\prime}\right) \forall[a, b] \in[I], \bar{R}(\widetilde{[a, b]})=}{[a, b] .} \\
& \Leftrightarrow(I L S E) \underline{R}(\widetilde{0})=\widetilde{0} . \\
& \Leftrightarrow(I U S E) \bar{R}(\widetilde{1})=\widetilde{1} .
\end{aligned}
$$


(2) $R$ is reflexive $\Leftrightarrow(I L R) \forall A \in F^{(i)}(U), \underline{R}(A) \subseteq A$. $\Leftrightarrow(I U R) \forall A \in F^{(i)}(U), A \subseteq \bar{R}(A)$.

(3) $R$ is symmetric $\Leftrightarrow \quad(I L S) \forall(x, y) \in U \times U$, $\underline{R}\left(\left(x_{\overline{1}}\right)^{c}\right)(y)=\underline{R}\left(\left(y_{\overline{1}}\right)^{c}\right)(x)$.

$$
\Leftrightarrow(I U S) \forall(x, y) \in U \times U, \bar{R}\left(x_{\overline{1}}\right)(y)=
$$
$\bar{R}\left(y_{\overline{1}}\right)(x)$.

(4) $R$ is transitive $\Leftrightarrow \quad(I L T) \forall A \in F^{(i)}(U), \underline{R}(A) \subseteq$ $\underline{R}(\underline{R}(A))$.

$$
\Leftrightarrow \frac{(I U T) \forall A \in F^{(i)}(U), \bar{R}(\bar{R}(A)) \subseteq}{\bar{R}(A) .}
$$

Proof. (1) By Theorem 9(3), (ILSE) and (IUSE) are equivalent and (ILSE ${ }^{\prime}$ ) and (IUSE ${ }^{\prime}$ ) are equivalent. We only need to prove that the serialisation of $R$ is equivalent to (IUSE) or (IUSE').

For any $[a, b] \in[I]$ and $x \in U$, we have

$$
\begin{aligned}
\bar{R}(\widetilde{[a, b]})(x) & =\bigvee_{y \in U}([a, b] \wedge R(x, y)) \\
& =[a, b] \wedge\left(\bigvee_{y \in U} R(x, y)\right) .
\end{aligned}
$$

(1) Assume that $R$ is serial. $\forall x \in U, \bigvee_{y \in U} R(x, y)=\overline{1}$. By $(\star)$,

$$
\bar{R}(\widetilde{[a, b]})(x)=[a, b] .
$$

Thus $\bar{R}(\widetilde{[a, b]})=\widetilde{[a, b]}$. $\forall x \in U$,

Conversely, assume $\bar{R}(\widetilde{[a, b]})=\widetilde{[a, b]}$ for each $[a, b] \in[I]$.

$$
\underline{R}(\widetilde{[a, b]})(x)=[a, b] .
$$

By $(\star), \bigvee_{y \in U} R(x, y) \geq[a, b]$.

Put $[a, b]=\overline{1}$. Then $\bigvee_{y \in U} R(x, y) \geq \overline{1}$.

This implies that $\bigvee_{y \in U} R(x, y)=\overline{1}$.

Thus $R$ is serial. $(\star)$,

(2) Assume that $R$ is serial. $\forall x \in U, \bigvee_{y \in U}(x, y)=\overline{1}$. By

$$
\bar{R}(\widetilde{[a, b]})(x)=[a, b] .
$$

Thus $\bar{R}(\widetilde{1})=\widetilde{1}$.

Conversely, assume that $\bar{R}(\widetilde{1})=\widetilde{1}$. By $(*), \forall x \in U$, $\bigvee_{y \in U} R(x, y)=\overline{1}$.

Thus $R$ is serial.

(2) By Theorem 9(3), (ILR) and (IUR) are equivalent. We only need to prove that the reflexivity of $R$ is equivalent to (IUR).

Assume that $R$ is reflexive. For any $A \in F^{(i)}(U)$ and $x \in U$, by the reflexivity of $R, R(x, x)=\overline{1}$. Then

$$
\begin{aligned}
(\bar{R}(A))(x) & =\bigvee_{y \in U}(A(y) \wedge R(x, y)) \\
& \geq A(x) \wedge R(x, x)=A(x) .
\end{aligned}
$$

Thus $A \subseteq \bar{R}(A)$.
Conversely, assume that (IUR) holds. $\forall x \in U$, pick $A=$ $x_{\overline{1}}$. By (IUR), $x_{\overline{1}} \subseteq \bar{R}\left(x_{\overline{1}}\right)$. By Remark 7(1),

$$
\overline{1}=x_{\overline{1}}(x) \leq \bar{R}\left(x_{\overline{1}}\right)(x)=R(x, x) \leq \overline{1} .
$$

This implies that $R(x, x)=\overline{1}$.

Thus $R$ is reflexive.

(3) By Theorem 9(3), (ILS) and (IUS) are equivalent. We only need to prove that the symmetry of $R$ is equivalent to (IUS).

$\forall x, y \in U$, by Remark $7(1)$,

$$
\bar{R}\left(y_{\overline{1}}\right)(x)=R(x, y), \quad \bar{R}\left(x_{\overline{1}}\right)(y)=R(y, x) .
$$

Thus the symmetry of $R$ is equivalent to (IUS).

(4) By Theorem 9(3), (ILT) and (IUT) are equivalent. We only need to prove that the transitivity of $R$ is equivalent to (IUT).

Assume that $R$ is transitive. $\forall x, y, z \in U$,

$$
R(x, z) \geq \bigvee_{y \in U}(R(x, y) \wedge R(y, z))
$$

Denote that $a_{x z}=\bigvee_{y \in U}(R(x, y) \wedge R(y, z))$. Then $\forall A \in$ $F^{(i)}(U), x \in U$,

$$
\begin{aligned}
\bar{R}(\bar{R}(A))(x) & =\bigvee_{y \in U}(\bar{R}(A)(y) \wedge R(x, y)) \\
& =\bigvee_{y \in U}\left(\left(\bigvee_{z \in U}(A(z) \wedge R(y, z))\right) \wedge R(x, y)\right) \\
& =\bigvee_{y \in U}\left(\bigvee_{z \in U}((A(z) \wedge R(y, z)) \wedge R(x, y))\right) \\
& =\bigvee_{y \in U}\left(\bigvee_{z \in U}(A(z) \wedge(R(y, z) \wedge R(x, y)))\right) \\
& \leq \bigvee_{y \in U}\left(\bigvee_{z \in U}\left(A(z) \wedge a_{x z}\right)\right)=\bigvee_{z \in U}\left(A(z) \wedge a_{x z}\right) \\
& \leq \bigvee_{z \in U}(A(z) \wedge R(x, z))=\bar{R}(A)(x) .
\end{aligned}
$$

Thus $\bar{R}(\bar{R}(A)) \subseteq \bar{R}(A)$.

Conversely, assume that (IUT) holds. $\forall x, y, z \in U$, by (IUT),

$$
\bar{R}\left(\bar{R}\left(z_{\overline{1}}\right)\right) \subseteq \bar{R}\left(z_{\overline{1}}\right) .
$$


By Remark 7(1),

$$
\begin{aligned}
R(x, y) \wedge R(y, z) & \leq \bigvee_{t \in U}(R(x, t) \wedge R(t, z)) \\
& =\bigvee_{t \in U}\left(R(x, t) \wedge \bar{R}\left(z_{\overline{1}}\right)(t)\right) \\
& =\bar{R}\left(\bar{R}\left(z_{\overline{1}}\right)\right)(x) \leq \bar{R}\left(z_{\overline{1}}\right)(x)=R(x, z)
\end{aligned}
$$

Thus $R$ is transitive.

Corollary 11. Let $(U, R)$ be an IVF approximation space. If $R$ is preorder, then

$$
\underline{R}(\underline{R}(A))=\underline{R}(A), \quad \bar{R}(\bar{R}(A))=\bar{R}(A) \quad\left(A \in F^{(i)}(U)\right) .
$$

Proof. This holds by Theorem 10.

\section{Axiomatic Characterizations of IVF Rough Approximation Operators}

In this section we show that rough approximation operators in IVF environment can be characterized by axioms; that is, we can find axiom sets of different IVF operators that guarantee the existence of different types of IVF relations which produce the same operators.

For any $\mathscr{A}_{J}=\left\{A_{j} \in F^{(i)}(U): j \in J\right\}, \mathscr{B}_{J}=\left\{B_{j} \in F^{(i)}(U)\right.$ : $j \in J\}$, we define the following:

$$
\mathscr{A}_{J} \odot \mathscr{B}_{J}=\bigcap_{j \in J}\left(A_{j} \cup B_{j}\right), \quad \mathscr{A}_{J} \odot \mathscr{B}_{J}=\bigcup_{j \in J}\left(A_{j} \cap B_{j}\right) .
$$

Denote that $\mathscr{I}_{J}=\left\{\widetilde{\left[a_{j}, b_{j}\right]}: j \in J\right\}$. We have

$$
\begin{gathered}
\mathscr{I}_{J} \odot \mathscr{A}_{J}=\bigcap_{j \in J}\left(\widetilde{\left[a_{j}, b_{j}\right]} \cup A_{j}\right), \\
\mathscr{I}_{J} \odot \mathscr{A}_{J}=\bigcup_{j \in J}\left(\left[a_{j}, b_{j}\right] A_{j}\right) .
\end{gathered}
$$

Let $H: F^{(i)}(U) \rightarrow F^{(i)}(U)$ be a mapping. Denote that

$$
H\left(\mathscr{A}_{J}\right)=\left\{H\left(A_{j}\right): A_{j} \in \mathscr{A}_{J}\right\} .
$$

Definition 12. Let $L, H: F^{(i)}(U) \rightarrow F^{(i)}(U)$ be two IVF operators. If

$$
(L(A))^{c}=H\left(A^{c}\right)\left(A \in F^{(i)}(U)\right),
$$

then $L$ and $H$ are called two dual IVF operators.

Remark 13. $L, H: F^{(i)}(U) \rightarrow F^{(i)}(U)$ are two dual IVF operators if and only if $(H(A))^{c}=L\left(A^{c}\right)$ for each $A \in F^{(i)}(U)$.
Theorem 14. Let $L, H: F^{(i)}(U) \rightarrow F^{(i)}(U)$ be two dual IVF operators. Then there exists an IVF relation $R$ on $U$ such that $L=\underline{R}$ and $H=\bar{R}$ if and only if $L$ satisfies the axioms (AL1) and $(A L 2)$ or, equivalently, $H$ satisfies the axioms (AU1) and (AU2):

(AL1) $\forall A \in F^{(i)}(U),[a, b], \in[I], L(\widetilde{[a, b]} \cup A)=\widetilde{[a, b]} \cup L(A)$;

(AL2) $\forall A, B \in F^{(i)}(U), L(A \cap B)=L(A) \cap L(B)$;

$(A U 1) \forall A \in F^{(i)}(U),[a, b] \in[I], H([a, b] A)=[a, b] H(A)$;

(AU2) $\forall A, B \in F^{(i)}(U), H(A \cup B)=H(A) \cup H(B)$.

Proof. Note that $L, H: F^{(i)}(U) \rightarrow F^{(i)}(U)$ are two dual IVF operators. Then (AL 1) and (AL2) are equivalent to (AU1) and (AU2). We only need to prove that $L=R$ and $H=\bar{R}$ if and only if $H$ satisfies the axioms (AU1) and (AU2).

Necessity. This holds by Theorem 9 .

Sufficiency. Assume that the operator $H$ satisfies the axioms (AU1) and (AU2). Define the following:

$$
R(x, y)=H\left(y_{\overline{1}}\right)(x) \quad(x, y \in U) .
$$

Let $A \in F^{(i)}(U)$. By Remark 4, $\forall x \in U$,

$$
\begin{aligned}
H(A)(x) & =\left(\bigcup_{y \in U}\left(A(y) y_{\overline{1}}\right)\right)(x) \\
& =\left(\bigcup_{y \in U} H\left(A(y) y_{\overline{1}}\right)\right)(x) \\
& =\left(\bigcup_{y \in U}\left(A(y) H\left(y_{\overline{1}}\right)\right)\right)(x) \\
& =\bigvee_{y \in U}\left(A(y) \wedge H\left(y_{\overline{1}}\right)(x)\right) \\
& =\bigvee_{y \in U}(A(y) \wedge R(x, y))=\bar{R}(A)(x) .
\end{aligned}
$$

Then $H(A)=\bar{R}(A)$. By Theorem 9(3),

$$
L(A)=\left(H\left(A^{c}\right)\right)^{c}=\left(\bar{R}\left(A^{c}\right)\right)^{c}=\underline{R}(A) .
$$

Thus $L=\underline{R}, H=\bar{R}$.

Corollary 15. Let $L, H: F^{(i)}(U) \rightarrow F^{(i)}(U)$ be two dual IVF operators. If $L$ satisfies the axioms (AL1) and (AL2) or, equivalently, $H$ satisfies the axioms (AU1) and (AU2), then $L$ satisfies the axiom $\left(A L 2^{\prime}\right)$ and $H$ satisfies the axiom $\left(A U 2^{\prime}\right)$ :

$\left(A L 2^{\prime}\right) \forall \mathscr{A}_{J} \subseteq F^{(i)}(U), L\left(\bigcap_{j \in J} A_{j}\right)=\bigcap_{j \in J} L\left(A_{j}\right) ;$ $\left(A U 2^{\prime}\right) \forall \mathscr{A}_{J} \subseteq F^{(i)}(U), H\left(\bigcup_{j \in J} A_{j}\right)=\bigcup_{j \in J} H\left(A_{j}\right)$.

Proof. This holds by Theorem 9 (4) and Theorem 14. 
Theorem 16. Let $L, H: F^{(i)}(U) \rightarrow F^{(i)}(U)$ be two dual IVF operators. Then there exists an IVF relation $R$ on $U$ such that $L=\underline{R}$ and $H=\bar{R}$, if and only if L satisfies the axiom (AL 3 ) or, equivalently, $H$ satisfies the axiom (AU3), where

$$
\begin{aligned}
(A L 3) \forall \mathscr{A}_{J} & \subseteq F^{(i)}(U), \mathscr{I}_{J} \subseteq[I],|J|=|U|, L\left(\mathscr{I}_{J} \odot \mathscr{A}_{J}\right)= \\
\mathscr{I}_{J} \odot L\left(A_{J}\right) ; &
\end{aligned}
$$

(AU3) $\forall \mathscr{A}_{J} \subseteq F^{(i)}(U), \mathscr{I}_{J} \subseteq[I],|J|=|U|, H\left(\mathscr{I}_{J} \odot \mathscr{A}_{J}\right)=I_{J} \odot$ $H\left(A_{J}\right)$.

Proof. Note that $L, H: F^{(i)}(U) \rightarrow F^{(i)}(U)$ are two dual IVF operators. Then (AL 3 ) is equivalent to (AU3). We only need to prove that $L=\underline{R}$ and $H=\bar{R}$ if and only if $H$ satisfies the axiom (AU3).

Necessity. Let $\forall \mathscr{A}_{J} \subseteq F^{(i)}(U), \mathscr{I}_{J} \subseteq[I],|J|=|U|$. By Theorem 9,

$$
\begin{aligned}
H\left(\mathscr{I}_{J} \odot \mathscr{A}_{J}\right) & =\bar{R}\left(\bigcup_{j \in J}\left([a, b] A_{j}\right)\right)=\bigcup_{j \in J} \bar{R}\left([a, b] A_{j}\right) \\
& =\bigcup_{j \in J}\left(\left[a_{j}, b_{j}\right] \bar{R}\left(A_{j}\right)\right) \\
& =\bigcup_{j \in J}\left(\left[a_{j}, b_{j}\right] H\left(A_{j}\right)\right)=\mathscr{I}_{J} \odot H\left(A_{J}\right) .
\end{aligned}
$$

Sufficiency. Assume that the operator $H$ satisfies the axiom (AU3). Define the following:

$$
R(x, y)=H\left(y_{\overline{1}}\right)(x) \quad(x, y \in U) .
$$

Let $A \in F^{(i)}(U)$. Pick $\mathscr{F}_{U}=\{\widetilde{A(y)}: y \in U\}$ and $\mathscr{P}_{U}=$ $\left\{y_{\overline{1}}: y \in U\right\}$. By Remark 4, then

$$
\begin{aligned}
H(A)(x) & =H\left(\bigcup_{y \in U}\left(A(y) y_{\overline{1}}\right)\right)(x) \\
& =H\left(\mathscr{I}_{U} \odot \mathscr{P}_{U}\right)(x)=\left(\mathscr{I}_{U} \odot H\left(\mathscr{P}_{U}\right)\right)(x) \\
& =\bigvee_{y \in U}\left(A(y) \wedge H\left(y_{\overline{1}}\right)(x)\right) \\
& =\bigvee_{y \in U}(A(y) \wedge R(x, y))=\bar{R}(A)(x)
\end{aligned}
$$

for each $x \in U$. Then $H(A)=\bar{R}(A)$.

Thus $H=\bar{R}$. By Theorem 9(3), $L(A)=\left(H\left(A^{c}\right)\right)^{c}=$ $\left(\bar{R}\left(A^{c}\right)\right)^{c}=\underline{R}(A)$.

Hence $L=R$.

Corollary 17. Let $L, H: F^{(i)}(U) \rightarrow F^{(i)}(U)$ be two dual IVF operators. If $L$ satisfies the axiom (AL3) or, equivalently, $H$ satisfies the axiom (AU3), then $L$ satisfies the axiom $\left(A L 3^{\prime}\right)$ and $H$ satisfies the axiom $\left(A U 3^{\prime}\right)$ :
$\left(A L 3^{\prime}\right) \forall \mathscr{A}_{J} \subseteq F^{(i)}(U), \mathscr{I}_{J} \subseteq[I], L\left(\mathscr{I}_{J} \odot \mathscr{A}_{J}\right)=\mathscr{I}_{J} \odot L\left(A_{J}\right) ;$ $\left(A U 3^{\prime}\right) \forall \mathscr{A}_{J} \subseteq F^{(i)}(U), \mathscr{I}_{J} \subseteq[I], H\left(\mathscr{I}_{J} \odot \mathscr{A}_{J}\right)=\mathscr{I}_{J} \odot H\left(A_{J}\right)$.

Proof. This holds by Theorems 9 and 16 .

The following results illustrate that IVF rough approximation operators generated by special IVF relations can be characterized by some axioms.

Theorem 18. Let $L, H: F^{(i)}(U) \rightarrow F^{(i)}(U)$ be two dual IVF operators. Then there exists a serial IVF relation $R$ on $U$ such that $L=R$ and $H=\bar{R}$ if and only if $L$ satisfies the axioms (AL1), (AL2), and (ILSE) or, equivalently, $H$ satisfies the axioms (AU1), (AU2), and (IUSE):

$(I L S E) L(\widetilde{0})=\widetilde{0}$,

(IUSE) $H(\widetilde{1})=\widetilde{1}$.

Proof. This holds by Theorems 10(1) and 14.

Corollary 19. Let $L, H \quad: \quad F^{(i)}(U) \rightarrow F^{(i)}(U)$ be dual operators. If $L$ satisfies the axioms (AL1), (AL2), and (ILSE) or, equivalently, $H$ satisfies the axioms (AU1), (AU2), and (IUSE), then L satisfies the axiom (ILSE') and H satisfies the axiom (IUSE'):

$\left(\operatorname{ILSE}^{\prime}\right) \forall[a, b] \in[I], L(\widetilde{[a, b]})=\widetilde{[a, b]} ;$

$\left(I U S E^{\prime}\right) \forall[a, b] \in[I], H(\widetilde{[a, b]})=\widetilde{[a, b]}$.

Proof. This holds by Theorems 10(1) and 18.

Theorem 20. Let $L, H: F^{(i)}(U) \rightarrow F^{(i)}(U)$ be two dual IVF operators. Then there exists a reflexive IVF relation $R$ on $U$ such that $L=\underline{R}$ and $H=\bar{R}$ if and only if $L$ satisfies the axioms ( $A L 1),(A L 2)$, and (ALR) or, equivalently, $H$ satisfies the axioms (AU1), (AU2), and (AUR):

$(A L R) \forall A \in F^{(i)}(U), L(A) \subseteq A$;

$(A U R) \forall A \in F^{(i)}(U), A \subseteq H(A)$.

Proof. This holds by Theorems 10(2) and 14

Theorem 21. Let $L, H: F^{(i)}(U) \rightarrow F^{(i)}(U)$ be two dual IVF operators. Then there exists a reflexive IVF relation $R$ on $U$ such that $L=R$ and $H=\bar{R}$ if and only if $L$ satisfies the axiom $\left(A L R^{\prime}\right)$ or, equivalently, $H$ satisfies the axiom $\left(A U R^{\prime}\right)$ :

$$
\begin{aligned}
&\left(A L R^{\prime}\right) \forall \mathscr{A}_{J} \subseteq F^{(i)}(U), \mathscr{I}_{J} \subseteq[I],|J|=|U|, \\
&\left(\mathscr{I}_{J} \odot \mathscr{A}_{J}\right) \cap\left(\mathscr{I}_{J} \odot L\left(A_{J}\right)\right)=L\left(\mathscr{I}_{J} \odot \mathscr{A}_{J}\right) ; \\
&\left(A U R^{\prime}\right) \forall \mathscr{A}_{J} \subseteq F^{(i)}(U), \mathscr{I}_{J} \subseteq[I],|J|=|U|, \\
&\left(\mathscr{I}_{J} \odot \mathscr{A}_{J}\right) \cup\left(\mathscr{I}_{J} \odot H\left(A_{J}\right)\right)=H\left(\mathscr{I}_{J} \odot \mathscr{A}_{J}\right) .
\end{aligned}
$$

Proof. Note that $H, L: F^{(i)}(U) \rightarrow F^{(i)}(U)$ are two dual IVF operators. Then $\left(\mathrm{ALR}^{\prime}\right)$ is equivalent to $\left(\mathrm{AUR}^{\prime}\right)$. We only need to prove that there exists a reflexive IVF relation $R$ on $U$ such 
that $L=\underline{R}$ and $H=\bar{R}$ if and only if $H$ satisfies the axiom $\left(\mathrm{AUR}^{\prime}\right)$.

Necessity. By Theorems 9 and 10(2),

$$
\begin{aligned}
& \left(\mathscr{I}_{J} \odot \mathscr{A}_{J}\right) \cup\left(\mathscr{I}_{J} \odot H\left(A_{J}\right)\right) \\
& =\left(\bigcup_{j \in J}\left(\left[a_{j}, b_{j}\right] A_{j}\right)\right) \cup\left(\bigcup_{j \in J}\left(\left[a_{j}, b_{j}\right] H\left(A_{i}\right)\right)\right) \\
& =\left(\bigcup_{j \in J}\left(\left[a_{j}, b_{j}\right] A_{i}\right)\right) \cup\left(\bigcup_{j \in J}\left(\left[a_{j}, b_{j}\right] \bar{R}\left(A_{i}\right)\right)\right) \\
& =\bigcup_{j \in J}\left(\left[a_{j}, b_{j}\right] \bar{R}\left(A_{i}\right)\right)=\bigcup_{j \in J} \bar{R}\left(\left[a_{j}, b_{j}\right] A_{i}\right) \\
& =\bar{R}\left(\bigcup_{j \in J}\left(\left[a_{j}, b_{j}\right] A_{i}\right)\right) \\
& =H\left(\bigcup_{j \in J}\left(\left[a_{j}, b_{j}\right] A_{j}\right)\right)=H\left(\mathscr{I}_{J} \odot \mathscr{A}_{J}\right) .
\end{aligned}
$$

Sufficiency. Let $A \in F^{(i)}(U)$. Pick $\mathscr{I}_{U}=\{\widetilde{A(y)}: y \in U\}$, $\mathscr{P}_{U}=\left\{y_{\overline{1}}: y \in U\right\}$. By Remark 4,

$$
\begin{aligned}
A & =\bigcup_{y \in U}\left(A(y) y_{\overline{1}}\right) \subseteq\left(\bigcup_{y \in U}\left(A(y) y_{\overline{1}}\right)\right) \\
& \cup\left(\bigcup_{y \in U}\left(A(y) H\left(y_{\overline{1}}\right)\right)\right) \\
& =\left(\mathscr{I}_{U} \odot \mathscr{P}_{U}\right) \cup\left(\mathscr{I}_{U} \odot H\left(\mathscr{P}_{U}\right)\right)=H\left(\mathscr{I}_{U} \odot \mathscr{P}_{U}\right) \\
& =H\left(\bigcup_{y \in U}\left(A(y) y_{\overline{1}}\right)\right)=H(A) .
\end{aligned}
$$

Then for any $\mathscr{A}_{J} \subseteq F^{(i)}(U)$ and $\mathscr{I}_{J} \subseteq[I]$ with $|J|=|U|$,

$$
\begin{aligned}
\mathscr{I}_{J} \odot \mathscr{A}_{J} & =\bigcup_{j \in J}\left(\left[a_{j}, b_{j}\right] A_{j}\right) \subseteq \bigcup_{j \in J}\left(\left[a_{j}, b_{j}\right] H\left(\mathscr{A}_{\mathrm{i}}\right)\right) \\
& =\mathscr{I}_{J} \odot H\left(\mathscr{A}_{J}\right) .
\end{aligned}
$$

So

$$
\begin{aligned}
\mathscr{I}_{J} \odot H\left(\mathscr{A}_{J}\right) & =\left(\mathscr{I}_{J} \odot \mathscr{A}_{J}\right) \cup\left(\mathscr{I}_{J} \odot H\left(\mathscr{A}_{J}\right)\right) \\
& =H\left(\mathscr{I}_{J} \odot \mathscr{A}_{J}\right) .
\end{aligned}
$$

By Theorem 16, there exists an IVF relation $R$ on $U$ such that $L=R$ and $H=\bar{R}$. Then $A \subseteq H(A)=\bar{R}(A)$. By Theorem 10(2), $R$ is reflexive.

Theorem 22. Let $L, H: F^{(i)}(U) \rightarrow F^{(i)}(U)$ be two dual IVF operators. Then there exists a symmetric IVF relation $R$ on $U$ such that $L=R$ and $H=\bar{R}$ if and only if $L$ satisfies the axioms (AL1), $(\bar{A} L 2)$, and (ALS) or, equivalently, $H$ satisfies the axioms (AU1), (AU2), and (AUS):
(ALS) $\forall x, y \in U, L\left(\left(x_{\overline{1}}\right)^{c}\right)(y)=L\left(\left(y_{\overline{1}}\right)^{c}\right)(x)$;

(AUS) $\forall x, y \in U, H\left(x_{\overline{1}}\right)(y)=H\left(y_{\overline{1}}\right)(x)$.

Proof. This holds by Theorems 10(3) and 14.

Theorem 23. Let $L, H: F^{(i)}(U) \rightarrow F^{(i)}(U)$ be two dual IVF operators. Then there exists a symmetric IVF relation $R$ on $U$ such that $L=\underline{R}$ and $H=\bar{R}$ if and only if $L$ satisfies the axiom $\left(A L R^{\prime}\right)$ or, equivalently, $H$ satisfies the axiom $\left(A U R^{\prime}\right)$ :

$\left(A L S^{\prime}\right) \forall A \in F^{(i)}(U), x \in U$,

$$
L\left(\bigcap_{y \in U}\left(\widetilde{A(y)} \cup\left(y_{\overline{1}}\right)^{c}\right)\right)(x)=\bigwedge_{y \in U}\left(A(y) \vee L\left(\left(x_{\overline{1}}\right)^{c}\right)(y)\right) ;
$$

$\left(A U S^{\prime}\right) \forall A \in F^{(i)}(U), x \in U$,

$$
H\left(\bigcup_{y \in U}\left(A(y) y_{\overline{1}}\right)\right)(x)=\bigvee_{y \in U}\left(A(y) \wedge H\left(x_{\overline{1}}\right)(y)\right) .
$$

Proof. Note that $L, H: F^{(i)}(U) \rightarrow F^{(i)}(U)$ are two dual IVF operators. Then $\left(\mathrm{ALS}^{\prime}\right)$ is equivalent to $\left(\mathrm{AUS}^{\prime}\right)$. We only need to prove that there exists a symmetric IVF relation $R$ on $U$ such that $L=\underline{R}$ and $H=\bar{R}$ if and only if $H$ satisfies the axiom $\left(\right.$ AUS $\left.^{\prime}\right)$.

Necessity. This holds by Theorems 9 and 10(3).

Sufficiency. Assume that $H$ satisfies the axiom $\left(\right.$ AUS $\left.^{\prime}\right)$. Define the following:

$$
R(x, y)=H\left(y_{\overline{1}}\right)(x) \quad(x, y \in U) .
$$

$\forall x, y \in U$,

$$
\begin{aligned}
R(y, x) & =H\left(x_{\overline{1}}\right)(y)=\bigvee_{z \in U}\left(y_{\overline{1}}(z) \wedge H\left(x_{\overline{1}}\right)(z)\right) \\
& =H\left(\bigcup_{z \in U}\left(y_{\overline{1}}(z) z_{\overline{1}}\right)\right)(x) \\
& =H\left(y_{\overline{1}}\right)(x)=R(x, y) .
\end{aligned}
$$

Then $R$ is symmetric.

Let $A \in F^{(i)}(U) . \forall x \in U$,

$$
\begin{aligned}
H(A)(x) & =H\left(\bigcup_{y \in U}\left(A(y) y_{\overline{1}}\right)\right)(x) \\
& =\bigvee_{y \in U}\left(A(y) \wedge H\left(x_{\overline{1}}\right)(y)\right) \\
& =\bigvee_{y \in U}(A(y) \wedge R(y, x)) \\
& =\bigvee_{y \in U}(A(y) \wedge R(x, y))=\bar{R}(A)(x) .
\end{aligned}
$$

This implies that $H(A)=\bar{R}(A)$.

Then $H=\bar{R}$. By Theorem 9(3), $L(A)=\underline{R}(A)$. 
Thus

$$
L=\underline{R} .
$$

Theorem 24. Let $L, H: F^{(i)}(U) \rightarrow F^{(i)}(U)$ be two dual $I V F$ operators. Then there exists a transitive IVF relation $R$ on $U$ such that $L=\underline{R}$ and $H=\bar{R}$ if and only if $L$ satisfies the axioms (AL1), $(A \bar{L} 2)$, and $(A L T)$ or, equivalently, $H$ satisfies the axioms (AU1), (AU2), and (AUT):

$(A L T) \forall A \in F^{(i)}(U), L(A) \subseteq L(L(A))$;

$(A U T) \forall A \in F^{(i)}(U), H(H(A)) \subseteq H(A)$.

Proof. This holds by Theorems 10(4) and 14.

Theorem 25. Let $L, H: F^{(i)}(U) \rightarrow F^{(i)}(U)$ be two dual IVF operators. Then there exists a transitive IVF relation $R$ on $U$ such that $L=\underline{R}$ and $H=\bar{R}$ if and only if $L$ satisfies the axiom $\left(A L T^{\prime}\right)$ or, equivalently, $H$ satisfies the axiom $\left(A U T^{\prime}\right)$ :

$\left(A L T^{\prime}\right) \forall \mathscr{A}_{J} \subseteq F^{(i)}(U), \mathscr{I}_{J} \subseteq[I],|J|=|U|$,

$$
\left(\mathscr{I}_{J} \odot L\left(A_{J}\right)\right) \cap\left(\mathscr{I}_{J} \odot L\left(L\left(A_{J}\right)\right)\right)=L\left(\mathscr{I}_{J} \odot \mathscr{A}_{J}\right) ;
$$

$$
\begin{aligned}
& \left(A U T^{\prime}\right) \forall \mathscr{A}_{J} \subseteq F^{(i)}(U), \mathscr{I}_{J} \subseteq[I],|J|=|U|, \\
& \quad\left(\mathscr{I}_{J} \odot H\left(\mathscr{A}_{J}\right)\right) \cup\left(\mathscr{I}_{J} \odot H\left(H\left(A_{J}\right)\right)\right)=H\left(\mathscr{I}_{J} \odot \mathscr{A}_{J}\right) .
\end{aligned}
$$

Proof. Note that $H, L: F^{(i)}(U) \rightarrow F^{(i)}(U)$ are two dual IVF operators. Then $\left(\mathrm{ALT}^{\prime}\right)$ is equivalent to $\left(\mathrm{AUT}^{\prime}\right)$. We only need to prove that there exists a transitive IVF relation $R$ on $U$ such that $L=R$ and $H=\bar{R}$ if and only if $H$ satisfies the axiom $\left(\mathrm{AUT}^{\prime}\right)$.

Necessity. By Theorems 9 and 10(4),

$$
\begin{aligned}
& \left(\mathscr{I}_{J} \odot H\left(\mathscr{A}_{J}\right)\right) \cup\left(\mathscr{I}_{J} \odot H\left(H\left(A_{J}\right)\right)\right) \\
& =\left(\bigcup_{j \in J}\left(\left[a_{j}, b_{j}\right] H\left(A_{i}\right)\right)\right) \cup\left(\bigcup_{j \in J}\left(\left[a_{j}, b_{j}\right] H\left(H\left(A_{i}\right)\right)\right)\right) \\
& =\left(\bigcup_{j \in J}\left(\left[a_{j}, b_{j}\right] \bar{R}\left(A_{i}\right)\right)\right) \cup\left(\bigcup_{j \in J}\left(\left[a_{j}, b_{j}\right] \bar{R}\left(\bar{R}\left(A_{i}\right)\right)\right)\right) \\
& =\bigcup_{j \in J}\left(\left[a_{j}, b_{j}\right] \bar{R}\left(A_{i}\right)\right)=\bigcup_{j \in J} \bar{R}\left(\left[a_{j}, b_{j}\right] A_{i}\right) \\
& =\bar{R}\left(\bigcup_{j \in J}\left(\left[a_{j}, b_{j}\right] A_{i}\right)\right) \\
& =H\left(\bigcup_{j \in J}\left(\left[a_{j}, b_{j}\right] A_{j}\right)\right)=H\left(\mathscr{I}_{J} \odot \mathscr{A}_{J}\right) .
\end{aligned}
$$

Sufficiency. Let $A \in F^{(i)}(U)$. Pick

$$
\mathscr{I}_{J}=\left\{\left[a_{j}, b_{j}\right]=\tilde{1}: j \in J\right\}, \quad \mathscr{A}=\left\{A_{j}=A: j \in J\right\} .
$$

Then

$$
\begin{aligned}
H(H(A))= & \mathscr{I}_{U} \odot H(H(\mathscr{A})) \\
& \subseteq\left(\mathscr{I}_{U} \odot H(\mathscr{A})\right) \cup\left(\mathscr{I}_{U} \odot H(H(\mathscr{A}))\right) \\
= & H\left(\mathscr{I}_{U} \odot \mathscr{A}\right)=H(A) .
\end{aligned}
$$

For any $\mathscr{A}_{J} \subseteq F^{(i)}(U)$ and $\mathscr{I}_{J} \subseteq[I]$ with $|J|=|U|$,

$$
\begin{aligned}
\mathscr{I}_{J} \odot H(H(\mathscr{A})) & =\bigcup_{j \in J}\left(\left[a_{j}, b_{j}\right] H\left(H\left(A_{j}\right)\right)\right) \\
& \subseteq \bigcup_{j \in J}\left(\left[a_{j}, b_{j}\right] H\left(A_{i}\right)\right)=\mathscr{I}_{J} \odot H\left(\mathscr{A}_{J}\right) .
\end{aligned}
$$

So

$$
\begin{aligned}
\mathscr{I}_{J} \odot H\left(\mathscr{A}_{J}\right) & =\left(\mathscr{I}_{J} \odot H\left(\mathscr{A}_{J}\right)\right) \cup\left(\mathscr{I}_{J} \odot H\left(H\left(\mathscr{A}_{J}\right)\right)\right) \\
& =H\left(\mathscr{I}_{J} \odot \mathscr{A}_{J}\right) .
\end{aligned}
$$

By Theorem 16, there exists an IVF relation $R$ on $U$ such that $L=\underline{R}$ and $H=\bar{R}$. So

$$
\bar{R}(\bar{R}(A))=H(H(A)) \subseteq H(A)=\bar{R}(A) .
$$

By Theorem 10(4), $R$ is transitive.

Theorem 26. Let $L, H: F^{(i)}(U) \rightarrow F^{(i)}(U)$ be two dual IVF operators. Then there exists a preorder IVF relation $R$ on $U$ such that $L=\underline{R}$ and $H=\bar{R}$ if and only if L satisfies the axiom (ALO) or, equivalently, $H$ satisfies the axiom (AUO):

$$
\begin{aligned}
& (A L O) \forall \mathscr{A}_{J} \subseteq F^{(i)}(U), \mathscr{I}_{J} \subseteq[I],|J|=|U|, \\
& \left(\mathscr{I}_{J} \odot A_{J}\right) \cap\left(\mathscr{I}_{J} \odot L\left(A_{J}\right)\right) \cap\left(\mathscr{I}_{J} \odot L\left(L\left(A_{J}\right)\right)\right) \\
& =L\left(\mathscr{I}_{J} \odot \mathscr{A}_{J}\right) ; \\
& (A U O) \forall \mathscr{A}_{J} \subseteq F^{(i)}(U), \mathscr{I}_{J} \subseteq[I],|J|=|U|, \\
& \left(\mathscr{I}_{J} \odot A_{J}\right) \cup\left(\mathscr{I}_{J} \odot H\left(\mathscr{A}_{J}\right)\right) \cup\left(\mathscr{I}_{J} \odot H\left(H\left(A_{J}\right)\right)\right) \\
& =H\left(\mathscr{I}_{J} \odot \mathscr{A}_{J}\right) .
\end{aligned}
$$

Proof. Note that $H, L: F^{(i)}(U) \rightarrow F^{(i)}(U)$ are two dual IVF operators. Then (ALO) is equivalent to (AUO). We only need to prove that there exists a preorder IVF relation $R$ on $U$ such 
that $L=\underline{R}$ and $H=\bar{R}$ if and only if $H$ satisfies the axiom (AUO).

Necessity. By Theorems 9 and 10, we have

$$
\begin{aligned}
& \left(\mathscr{I}_{J} \odot A_{J}\right) \cup\left(\mathscr{I}_{J} \odot H\left(\mathscr{A}_{J}\right)\right) \cup\left(\mathscr{I}_{J} \odot H\left(H\left(A_{J}\right)\right)\right) \\
& =\left(\bigcap_{j \in J}\left(\widetilde{\left[a_{j}, b_{j}\right]} \cup L\left(A_{j}\right)\right)\right) \cap\left(\bigcap_{j \in J}\left(\widetilde{\left[a_{j}, b_{j}\right]} \cup L\left(L\left(A_{j}\right)\right)\right)\right) \\
& =\left(\bigcup_{j \in J}\left(\left[a_{j}, b_{j}\right] A_{i}\right)\right) \cup\left(\bigcup_{j \in J}\left(\left[a_{j}, b_{j}\right] \bar{R}\left(A_{i}\right)\right)\right) \\
& \cup\left(\bigcup_{j \in J}\left(\left[a_{j}, b_{j}\right] \bar{R}\left(\bar{R}\left(A_{i}\right)\right)\right)\right) \\
& =\bigcup_{j \in J}\left(\left[a_{j}, b_{j}\right] \bar{R}\left(A_{i}\right)\right)=\bigcup_{j \in J} \bar{R}\left(\left[a_{j}, b_{j}\right] A_{i}\right) \\
& =\bar{R}\left(\bigcup_{j \in J}\left(\left[a_{j}, b_{j}\right] A_{i}\right)\right) \\
& =H\left(\bigcup_{j \in J}\left(\left[a_{j}, b_{j}\right] A_{j}\right)\right)=H\left(\mathscr{I}_{J}^{\odot} \mathscr{A}_{J}\right) .
\end{aligned}
$$

Sufficiency. Let $A \in F^{(i)}(U)$. Pick

$$
\mathscr{I}_{U}=\{A(y): y \in U\}, \quad \mathscr{P}_{U}=\left\{y_{\overline{1}}: y \in U\right\} .
$$

By Remark 4,

$$
\begin{aligned}
A & =\bigcup_{y \in U}\left(A(y) y_{\overline{1}}\right) \subseteq\left(\bigcup_{y \in U}\left(A(y) y_{\overline{1}}\right)\right) \\
& \cup\left(\bigcup_{y \in U}\left(A(y) H\left(y_{\overline{1}}\right)\right)\right) \cup\left(\bigcup_{y \in U}\left(A(y) H\left(H\left(y_{\overline{1}}\right)\right)\right)\right) \\
& =\left(\mathscr{I}_{U} \odot \mathscr{P}_{U}\right) \cup\left(\mathscr{I}_{U} \odot H\left(\mathscr{P}_{U}\right)\right) \cup\left(\mathscr{I}_{U} \odot H\left(H\left(\mathscr{P}_{U}\right)\right)\right) \\
& =H\left(\mathscr{I}_{U} \odot \mathscr{P}_{U}\right) \\
& =H\left(\bigcup_{y \in U}\left(A(y) y_{\overline{1}}\right)\right)=H(A) .
\end{aligned}
$$

For any $\mathscr{A}_{J} \subseteq F^{(i)}(U)$ and $\mathscr{I}_{J} \subseteq[I]$ with $|J|=|U|$,

$$
\begin{aligned}
\mathscr{I}_{J} \odot \mathscr{A}_{J} & =\bigcup_{j \in J}\left(\left[a_{j}, b_{j}\right] A_{j}\right) \\
& \subseteq \bigcup_{j \in J}\left(\left[a_{j}, b_{j}\right] H\left(A_{i}\right)\right) \\
& =\mathscr{I}_{J} \odot H\left(\mathscr{A}_{J}\right) .
\end{aligned}
$$

Pick

$$
\mathscr{I}_{J}=\left\{\left[a_{j}, b_{j}\right]=\widetilde{1}: j \in J\right\}, \quad \mathscr{A}=\left\{A_{j}=A: j \in J\right\} .
$$

Then

$$
\begin{aligned}
H(H(A)) & =\mathscr{I}_{U} \odot H(H(\mathscr{A})) \subseteq\left(\mathscr{I}_{U} \odot \mathscr{A}\right) \\
& \cup\left(\mathscr{I}_{U} \odot H(\mathscr{A})\right) \cup\left(\mathscr{I}_{U} \odot H(H(\mathscr{A}))\right) \\
& =H\left(\mathscr{I}_{U} \odot \mathscr{A}\right)=H(A) .
\end{aligned}
$$

So

$$
\begin{aligned}
\mathscr{I}_{J} \odot H(H(\mathscr{A})) & =\bigcup_{j \in J}\left(\left[a_{j}, b_{j}\right] H\left(H\left(A_{j}\right)\right)\right) \\
& \subseteq \bigcup_{j \in J}\left(\left[a_{j}, b_{j}\right] H\left(A_{i}\right)\right) \\
& =\mathscr{I}_{J} \odot H\left(\mathscr{A}_{J}\right) .
\end{aligned}
$$

Thus

$$
\begin{aligned}
\mathscr{I}_{J} \odot H\left(\mathscr{A}_{J}\right) & =\left(\mathscr{I}_{J} \odot \mathscr{A}_{J}\right) \cup\left(\mathscr{I}_{J} \odot H\left(\mathscr{A}_{J}\right)\right) \\
& \cup\left(\mathscr{I}_{J} \odot H\left(H\left(\mathscr{A}_{J}\right)\right)\right) \\
& =H\left(\mathscr{I}_{J} \odot \mathscr{A}_{J}\right) .
\end{aligned}
$$

By Theorem 16, there exists an IVF relation $R$ on $U$ such that $L=\underline{R}$ and $H=\bar{R}$. Then

$$
\begin{gathered}
A \subseteq H(A)=\bar{R}(A), \\
\bar{R}(\bar{R}(A))=H(H(A)) \subseteq H(A)=\bar{R}(A) .
\end{gathered}
$$

By Theorem 10, $R$ is preorder.

\section{Conclusions}

In this paper, we have obtained axiomatic characterizations of IVF rough approximation operators; we can find axiom sets of different IVF operators that guarantee the existence of different types of IVF relations which produce the same operators. We can consider some engineering applications of our proposed notions and theories. In the future, we will study these problems.

\section{Conflict of Interests}

The author declares that there is no conflict of interests regarding the publication of this paper.

\section{Acknowledgment}

This work is supported by the Natural Science Foundation of Guangxi (no. 2012GXNSFDA276040). 


\section{References}

[1] Z. Pawlak, "Rough sets," International Journal of Computer and Information Sciences, vol. 11, no. 5, pp. 341-356, 1982.

[2] Z. Pawlak, Rough Sets: Theoretical Aspects of Reasoning About Data, Kluwer Academic Publishers, Dordrecht, The Netherlands, 1991.

[3] M. B. Gorzałczany, "Interval-valued fuzzy controller based on verbal model of object," Fuzzy Sets and Systems, vol. 28, no. 1, pp. 45-53, 1988.

[4] I. B. Türksen, "Interval valued fuzzy sets based on normal forms," Fuzzy Sets and Systems, vol. 20, no. 2, pp. 191-210, 1986.

[5] B. Sun, Z. Gong, and D. Chen, "Fuzzy rough set theory for the interval-valued fuzzy information systems," Information Sciences, vol. 178, no. 13, pp. 2794-2815, 2008.

[6] Z. Gong, B. Sun, and D. Chen, "Rough set theory for the interval-valued fuzzy information systems," Information Sciences, vol. 178, no. 8, pp. 1968-1985, 2008.

[7] H.-Y. Zhang, W.-X. Zhang, and W.-Z. Wu, "On characterization of generalized interval-valued fuzzy rough sets on two universes of discourse," International Journal of Approximate Reasoning, vol. 51, no. 1, pp. 56-70, 2009.

[8] T. K. Mondal and S. K. Samanta, "Topology of interval-valued fuzzy sets," Indian Journal of Pure and Applied Mathematics, vol. 30, no. 1, pp. 23-38, 1999.

[9] H. Lai and D. Zhang, "Fuzzy preorder and fuzzy topology," Fuzzy Sets and Systems, vol. 157, no. 14, pp. 1865-1885, 2006. 


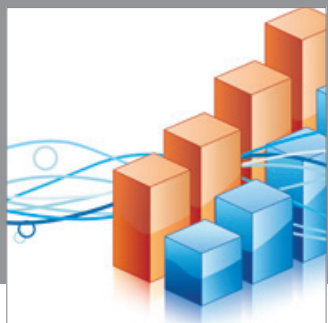

Advances in

Operations Research

mansans

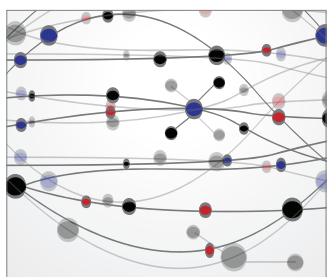

The Scientific World Journal
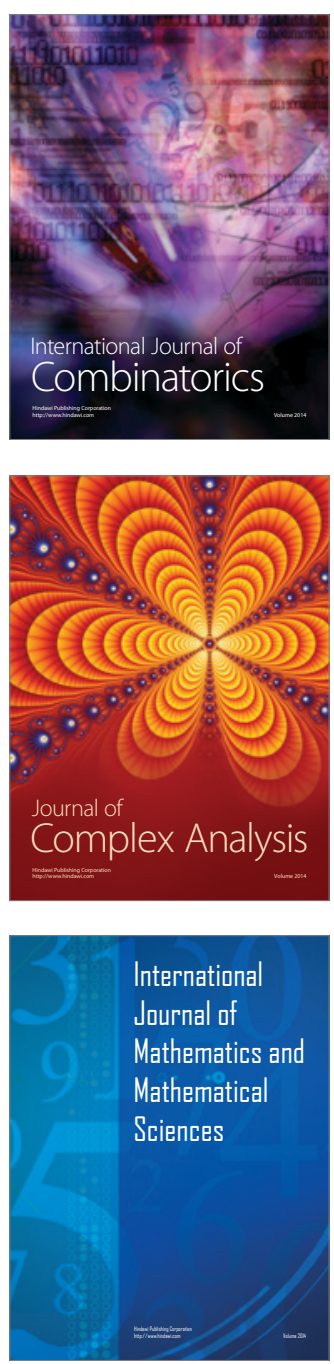
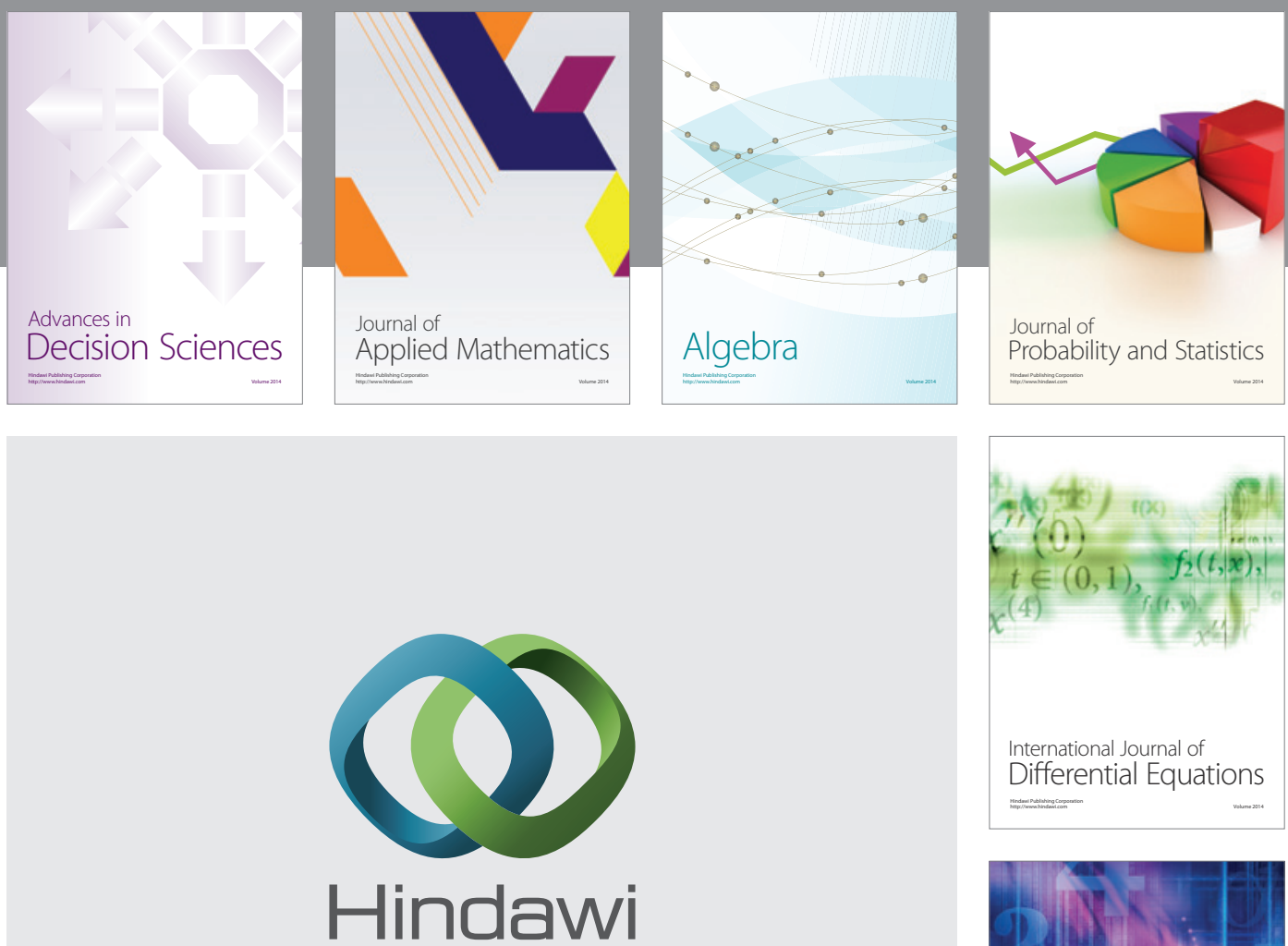

Submit your manuscripts at http://www.hindawi.com
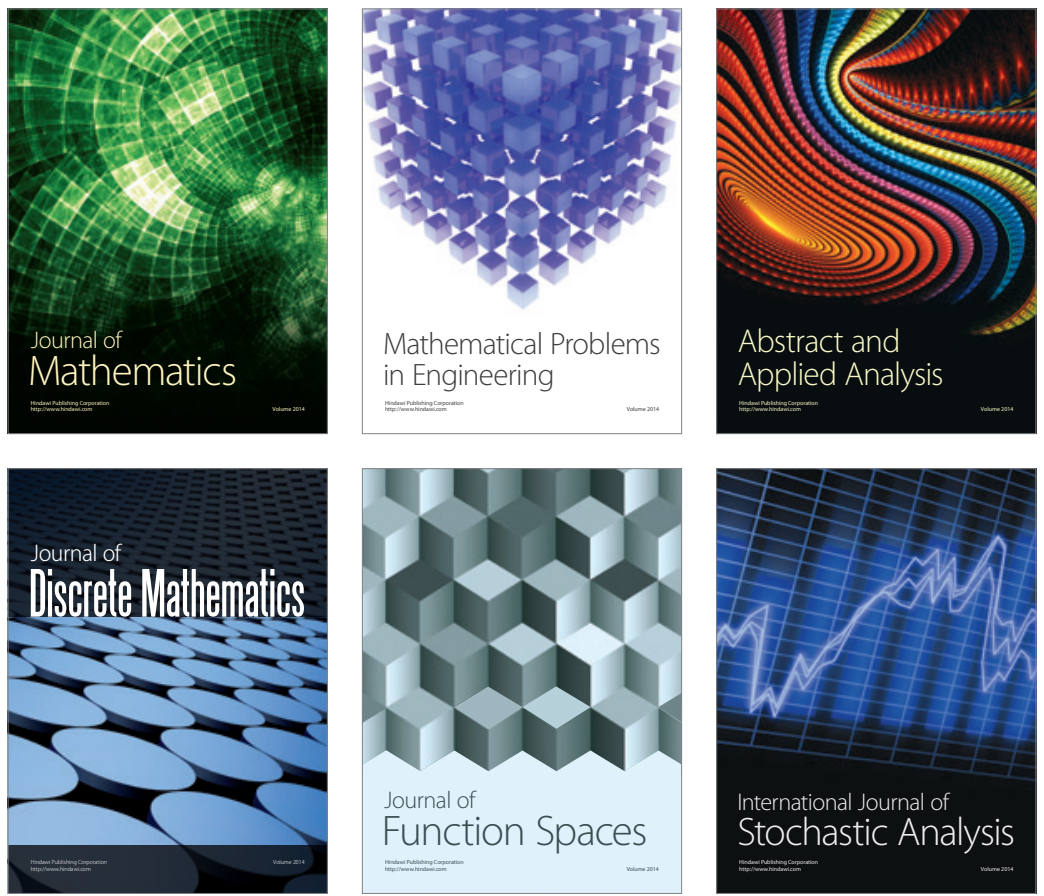

Journal of

Function Spaces

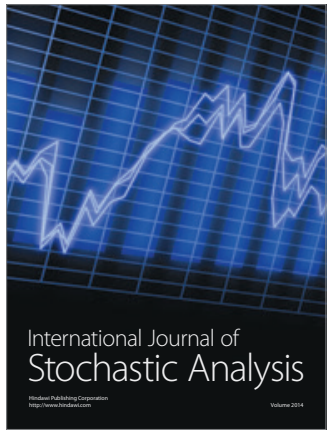

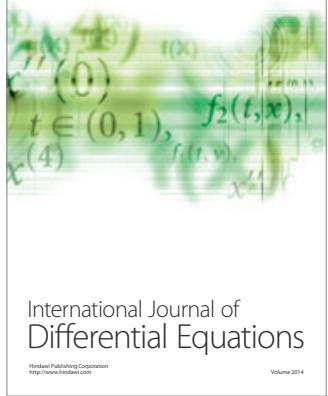
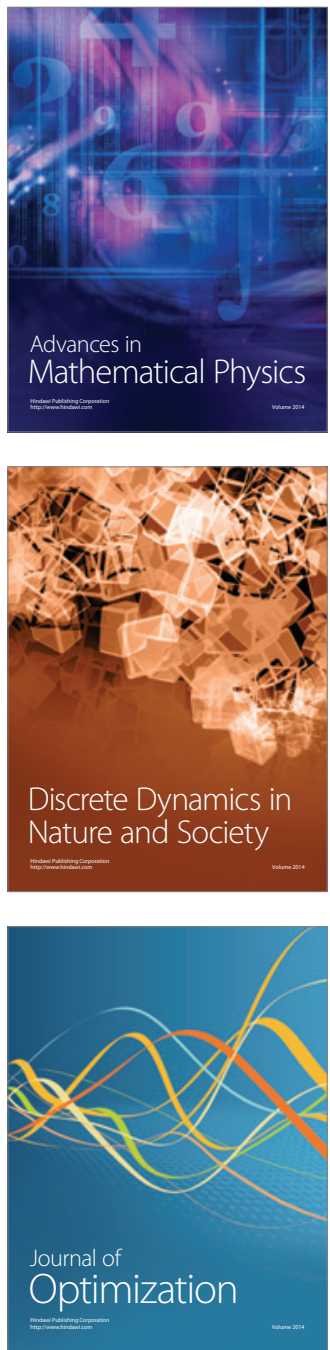